\title{
A Field Study of Teachers Using a Curriculum-integrated Digital Game
}

\author{
Zhongxiu Peddycord-Liu, Veronica Cateté, Jessica Vandenberg, Tiffany Barnes, Collin F. Lynch, \\ Teomara Rutherford \\ North Carolina State University \\ aurora.peddycord-liu@sas.com,vmcatete,jvanden2,tmbarnes,cflynch,taruther@ncsu.edu
}

\begin{abstract}
We present a new framework describing how teachers use ST Math, a curriculum-integrated, year-long educational game, in 3rd-4th grade classrooms. We combined authentic classroom observations with teacher interviews to identify teacher needs and practices. Our findings extended and contrasted with prior work on teachers' behaviors around classroom games, identifying differences likely arising from a digital platform and year-long curricular integration. We suggest practical ways that curriculum-integrated games can be designed to help teachers support effective classroom culture and practice.
\end{abstract}

\section{CCS CONCEPTS}

- Applied computing $\rightarrow$ Education; Computer games.

\section{KEYWORDS}

Educational Games; K12; Classroom Integration; Teachers

\section{ACM Reference Format:}

Zhongxiu Peddycord-Liu, Veronica Cateté, Jessica Vandenberg, Tiffany Barnes, Collin F. Lynch,, Teomara Rutherford. 2019. A Field Study of Teachers Using a Curriculum-integrated Digital Game. In CHI Conference on Human Factors in Computing Systems Proceedings (CHI 2019), May 4-9, 2019, Glasgow, Scotland, UK. ACM, New York, NY, USA, 12 pages. https://doi.org/10.1145/3290605.3300658

\section{INTRODUCTION}

Educational games can positively impact student learning $[4,9,26]$ and are becoming increasingly popular in classrooms worldwide [1,52], with curriculum-integrated games identified as a key market demand [41, 53]. However, more

Permission to make digital or hard copies of all or part of this work for personal or classroom use is granted without fee provided that copies are not made or distributed for profit or commercial advantage and that copies bear this notice and the full citation on the first page. Copyrights for components of this work owned by others than ACM must be honored. Abstracting with credit is permitted. To copy otherwise, or republish, to post on servers or to redistribute to lists, requires prior specific permission and/or a fee. Request permissions from permissions@acm.org.

CHI 2019, May 4-9, 2019, Glasgow, Scotland, UK

() 2019 Association for Computing Machinery.

ACM ISBN 978-1-4503-5970-2/19/05 ..\$15.00

https://doi.org/10.1145/3290605.3300658 research is needed on how best to integrate games into real-world classrooms. One recent literature review concluded that the crucial role of teachers has been neglected or marginalized, in both research and in game design [33], with most teacher-focused educational games research focusing on barriers to adoption $[12,14,18,30,32,33,50]$. Only a few studies have focused on the pedagogical practices around educational games in authentic classrooms (e.g., [18, 24, 34, 57]), especially for curriculum-integrated games $[11,18]$. There is a critical need to understand how games are used in classrooms, and to use this understanding to inform game design and benefit student learning.

We study the use of a curriculum-integrated digital game in the classrooms of eight teachers with varied game experience levels. We combined field observations with semistructured interviews to explore teacher use in detail and derive suggestions for game design. We were specifically interested in how teachers integrated and orchestrated ST Math in their classrooms and teaching practice.

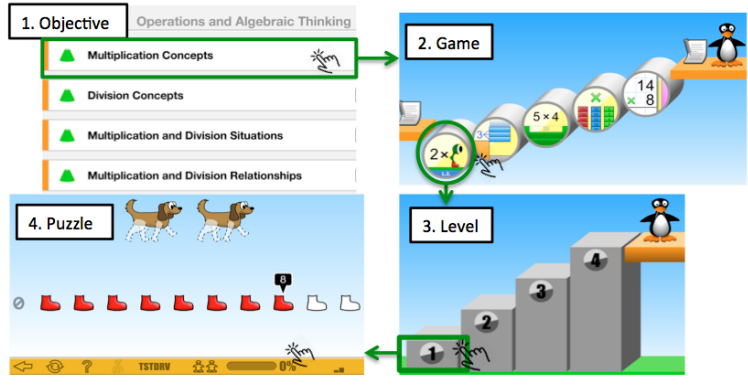

Figure 1: Screenshots of ST Math Content Hierarchy

Spatial Temporal Math (www.stmath.com), designed by the MIND Research Institute, helps students learn K-12 math concepts through helping Jiji the penguin solve puzzles (Figure1). ST Math is similar to other drill-and-practice standardsaligned mini-games such as Astra Eagle [25], Motion Math [42] and Math Garden [55]. ST Math was specifically designed to play alongside schools' existing curricula, and is currently used by more than a thousand schools in the U.S. We have a longstanding collaboration with MIND, who provided us with game logs and game design information. Studying ST Math provides a unique opportunity to gain insight 
into classroom use of a large-scale curriculum-integrated game, potentially benefiting thousands of students.

ST Math teaches through visualizations and spatial reasoning, minimizing written instructions. Every student answer leads to animated feedback. For example, one ST Math puzzle practices multiplying $3^{*} 4$ by showing 3 dogs with missing shoes. Students answer with the number of shoes needed; animated feedback will show shoes missing for answers less than 12 , the right number of shoes for an answer of 12 , or how many shoes are left for answers more than 12. Students are supposed to learn and correct their answers based on the feedback, consequently developing their understanding of multiplication. Students may struggle to get the answer, but the process itself (i.e., spatial-reasoning and reflection on wrong answers) develops skills and a mindset-productive struggle is a key component of ST Math.

ST Math uses a tiered structure with top level objectives (math concepts), then games (problem solving scenarios), levels (progressing difficulties or puzzle groups), and puzzles (specific questions). Each grade has around 20-30 objectives and hundreds of levels to be played throughout the academic year. Each objective starts with a pre-test and ends with a post-test of multiple-choice questions. Students must complete the games and levels in a predetermined order. Each level must be completed within a set number of Jiji's lives. Each wrong answer costs one life, and students must reattempt the whole level if all lives are exhausted.

\section{LITERATURE REVIEW}

Curriculum-integrated games in this paper are games with content aligned to academic standards, intended to be used all year long as a supplement to schools' existing curricula. Therefore, curriculum integrated games often contain a large collection of content and are intended to be played throughout the semester, many being embodied into existing classroom activities. Seven among the $34 \mathrm{~K} 12$ math games reviewed in [38] have content aligned to academic standarsd, such as Astra Eagle [25], Motion Math [42], VMathLive [28], and ST Math [45]. Most studies have shown that such games significantly improved standardized math test scores $[25,28,42,45,51]$.

Controlled experiments have suggested that digital games are more effective when combined with structured classroom activities, such as debriefing and bridging $[6,11,20,44]$. For example, Rowe et al. found that students learned better when the teacher bridged game-based learning to classroom learning using game examples and discussion [44]. Because curriculum-integrated games are designed to enhance existing curricula, integrating them with classroom teaching is essential to their effectiveness.

Meanwhile, using games in classrooms is practically challenging for teachers. Dillenbroug and Jermann argued that teachers who 'orchestrate' the classroom and its technologies are constantly facing multi-constraint management problems [17]. Common challenges include lack of time [12, 14, 18, $30,32,33,48,50]$, connecting games with learning objectives $[11,14,18,33,58]$, identifying teachable moments during gameplay [12,33], and classroom management $[14,58]$. Such challenges may become more critical for games such as ST Math, that are built to be played more frequently and for the whole academic year, and in alignment with the curriculum.

Only a limited number of studies have investigated the practices surrounding the use of specific digital games in authentic classroom settings, let alone curriculum-integrated games. In a case study of one teacher, Watson et al. identified three teacher strategies: learning by playing the game; setting goals for gameplay and encouraging reflection; and identifying teachable moments during gameplay [57]. From three teacher case-studies, Eastwood and Sadler found that teachers viewed and used curriculum-integrated materials differently, demonstrating the importance of flexible and adaptive design [18]. Nanavati et al. found that games caused some shifts of authority from teachers to students, and identified disconnects between the games' purposes and how they were used in context [34]. Callaghan et al. surveyed ST Math teachers and reported that they needed the most help with assisting struggling students, being aware of the game's purpose and the underlying mathematics in later game levels, and better aligning games with the curriculum [11].

Our approach is informed by Kangas et al's research on teachers' pedagogical activities surrounding digital and nondigital games [24]. Through their review of 35 studies, Kangas et al. derived five categories of teacher usage that should be intentionally supported [24]: 1) planning: creating a pedagogical frame for game-based learning, such as situating the game; 2) orientation: introducing the game and background concepts before play; 3) playing: tutoring students' gameplay; 4) elaborating: leading discussion after gameplay; and 5) reflecting: teacher's self-reflection process. However, as Kangas et al. pointed out, the papers they reviewed documented teacher activities in varied levels of detail, had very different study designs, and many researched games that were only played a few times in the classroom. Thus, there is a need to investigate how teachers use long-term, curriculum-integrated games in authentic classroom settings, to understand how their practice may differ from games used for shorter durations, and which may not have been designed to align with a specific curriculum or standards.

\section{METHOD}

We focused on one school district in the southern U.S., where ST Math is a mandatory intervention. Principals at all 81 elementary schools were invited to participate; seven volunteered within a two-week recruitment period. All 3rd and 4th 
grade teachers from these schools were invited to complete a screening survey. Eight female teachers from six schools responded to and participated. These schools had over $44 \%$ students eligible for free/reduced lunch and five teachers' classes had $100 \%$ eligibility. Teachers' experience with ST Math fell into three levels, High (H1-4), those who have been teaching with ST Math since it was first introduced in the district over six years ago, Moderate (M1-2), those using ST Math for 1-3 years, and Low (L1-2), those using ST Math for less than a year. L1 recently transferred to the county and L2 was a first year teacher. We did not collect other demographic information from the observed classes.

We took a grounded theory case-study approach to understand the teachers' experiences, specifically focusing on their use of ST Math's teacher features and their classroom integration of ST Math. The first and last authors have had years of experience researching ST Math and experience playing the game as students, manipulating the game as teachers, and viewing teacher resources. The remaining authors and field observers come from education and computer science, with varied experience in educational research, learning technology design, and K-12 outreach. The third author is an experienced teacher of elementary students.

Initial classroom observations were followed by a teacher interview and subsequent observations. We conducted 43 observations, with 2 teachers being observed 4 times, 1 teacher with 5 observations, and 5 teachers with 6 . Classroom observations were conducted in teachers' regular math sessions with planned ST Math use. We ensured that these observations covered diverse scenarios of using ST Math both in class and in lab. Each observation lasted from 20 minutes up to an hour, depending on class schedules. The observers were trained on the classroom observation protocol to improve consistency between observers. In most observation sessions, we assigned one observer to focus on the teacher and the remaining focused on students. The teacher observer took field notes on the classroom set-up, the major instructional activities, and detailed all of the interactions between students and teachers during the ST Math session. The student observers recorded student affect and behaviors and any interactions of note. After each session, observers conferred and noted interesting results, relating them to prior observations, literature, and theory. We rotated observers for the same teacher to facilitate diverse perspectives.

We conducted semi-structured teacher interviews after several observations to facilitate a meaningful conversation around the teacher's perspective and classroom context. Each interview lasted 30-50 minutes after school, during which we incorporated questions based on field observations with field observers present. The first author observed at least one session of each class format for each teacher before conducting interviews. We informed teachers of the anonymization practice and that researchers were not ST Math stakeholders. During the interview, we asked teachers about their activities and rationales before, during, and after the use of ST Math in the classroom, their perceptions of the students' use of ST Math, and their suggestions to improve the system. The semistructured interview facilitated a guided discussion where the interviewer could help teachers expand their thoughts and reflect on their decision-making. The interviews were audio-recorded with strategic note taking to help with later interpretations; audio recordings were transcribed verbatim.

After field observations and interviews, we conducted grounded theory analyses of teacher interviews following Saldana's two round coding method, where the first round is used to identify and describe codes, and the second round is to identify common patterns [49]. We carefully used our field notes and experiences to understand the context for each interview. The first author, who was also the interviewer and primary teacher observer, compiled and verbally summarized the field notes to establish the context, evaluate teacher statements, and facilitate discussion between the two coders. Initially, the first and second authors coded two interviews independently, using four types of coding as described by Saldana: structural coding to relate data to research questions, such as 'teacher practices' and 'teacher suggestions'; process coding to identify activities and actions, such as 'offering affective help, descriptive coding to summarize statements and identify involved factors, such as 'time management'; and evaluation coding to describe teachers' comments for ST Math developers, such as 'ST Math report: need live feedback.' Then, the researchers collaborated to refine codes into a codebook. Next, each coder independently coded three different interviews, checked each other's codes, and then met to discuss and collaboratively update the codebook. The coders then collaboratively conducted pattern coding [49] to identify developed themes and patterns across interviews.

\section{HOW WERE TEACHERS USING ST MATH?}

From classroom observations and interviews, we identified four types of activities related to teacher's classroom use of ST Math: Preparation, Integration, Intervention and Datainformed Practice. In Table 1, we have aligned these with activities identified in Kangas et al.'s literature review on teacher activities surrounding digital and non-digital games [24]. Our work both extends and contrasts with Kangas et al.'s game-based learning framework; differences likely arise from our focus on a school-year-long curriculum-integrated digital game, rather than shorter games whose content may not directly support curricular standards. In the following sections, we describe each type of teacher activity, connect these activities to prior literature, and recommend game designs that could better support teachers and students using curriculum-integrated digital games. 
Table 1: Teacher Activities to Orchestrate a CurriculumAligned Digital Game in Classrooms.

\begin{tabular}{|l|l|}
\hline Preparation & Integration \\
- Observe Student Gameplay & - Set up for Classroom Formats [planning] \\
- Use Online Resources & - Foster Gameplay Culture [planning] \\
- Self Gameplay & - Order the Curriculum [planning] \\
- Attend Professional Development & - Connect Game Content with Classroom \\
- Communicate with Peer Teachers & Content [planning, orientation, elaboration] \\
\hline Intervention & Data-informed Practice \\
- Cognitive Intervention [playing] & - Identify Struggling Students Combining in- \\
- Affective Intervention & and out-of-game Assessments \\
- Organize Peer Tutors [playing] & - Identify Math Content to Review/Reteach \\
- Progress Management [playing] & - Communicate with Students [elaboration] \\
\hline
\end{tabular}

\subsection{Preparation}

Preparation is the work that teachers do to get ready to use a curriculum-integrated game. In this category, teachers themselves learn about ST Math to prepare for using it in classrooms. This preparation category was not explicitly identified by Kangas et al. [24]. We identified five major activities in this category: observing students' gameplay, using online resources, playing the game themselves, communicating with peer teachers, and attending professional development (PD). All teachers stated that they relied heavily upon observing students' gameplay to learn the game. This is supported by our classroom observations, showing that many teachers asked students to explain what they were doing as students played. Three highly-experienced teachers also learned by playing the game, with H3 in charge of ST Math at her school, and $\mathrm{H} 1$ and $\mathrm{H} 2$ playing ST Math with their children at home. The remaining teachers stated that they relied mostly on figuring it out during students' gameplay. $\mathrm{H} 1$ and $\mathrm{H} 4$ mentioned that having previous experience using ST Math helped them accumulate knowledge across years.

Teachers who recently attended PD (M1-2, L2) commented that the required county PD was too basic. For example, M1 stated that the county PD was primarily about "how the game looks," with "very little about the reports, or the teacher information that's on there." L2 noted that she played the game during PD but didn't know "how to manipulate [it]" or "why we use it." L1 recently transferred into the district and missed the PD. Meanwhile, more experienced teachers stated that MIND's supplemental online resources (accessible from a 'ST Math Central' button) were effective, but new teachers L1 and L2 were not aware that this resource existed.

4.1.1 Design Implications. Our results support prior findings that both teachers and students continuously help each other in understanding and using the game [34]. This could be especially true for games with large amounts of content that are intended to be played over a long period of time. Based on teachers' comments on PD, a prior ST Math study [11], and a literature review of broader ICT training [10], we suggest that PD for such games should focus more on the game's purpose and integration practices, instead of specific interface elements that can be learned from observing students. Moreover, as the two new teachers expressed ignorance regarding the 'ST Math Central' button, it may be helpful to have other mechanisms to help teachers notice where to find and access PD resources. Our results also reiterate Girotto's recommendation that teacher preparation should be integrated into their existing work-flow with educational software, providing teachers just-in-time information needed to make decisions [19]. For example, the software could require that teachers watch mandatory short videos as soon as they register in ST Math.

\subsection{Integration}

Integration is the work that teachers do to fit the the game into the classroom. Our Integration category closely aligns with Kangas et al.'s planning category, where teachers create a pedagogical frame to prepare students to learn, organize gameplay situations, and fit the game into the curriculum [24]. Based on our analyses, the teachers conducted the following Integration activities: organize gameplay into different class formats, foster a gameplay culture, reorder ST Math objectives, and connect the game content with math content. The last activity is also related to Kangas et al's orientation and elaboration phases, but we did not observe these activities directly before or after the gameplay sessions.

4.2.1 Class Format. We observed three class formats that each appeared to significantly impact gameplay interaction: labseating (18 observations, 6 teachers), free-seating ( 9 observations, 3 teachers), and rotation-seating (16 observations, 5 teachers). In lab-seating, all students played ST Math during the whole class, by sitting at designated spots in a lab-like environment. Teachers could clearly see students' screens, and were able to pay closer attention to ST Math gameplay as compared to other formats. In free-seating, students played ST Math the whole time, but were free to move around the classroom, sitting as individuals or in groups, using tablets or laptops. Student-to-student interactions were more frequent, and teachers had to move around continuously to see all students' screens. Consequently, students who sat by themselves, sat in corners, or were reluctant to seek teachers' help, were often ignored. In rotation-seating, the students were split into groups with one group doing ST Math while the other group(s) did other activities, such as engaging in teacher-led instruction. Students rotated groups after a set time. In this format, the teachers were often busy with students doing other activities, and the "self-sufficient" ST Math groups rarely received attention. Our interviews made clear that the class formats were influenced by school policy, scheduling, and the availability of technologies in classrooms. 
In interviews, teachers reflected on the pros and cons of different class formats. M1 and L1 liked that the rotationseating allowed them time to focus on students who needed help by putting the rest on ST Math. H2, H4, and L2 believed that students got more chances to work with each other when left on their own. For lab- and free-seatings, M2 liked that students got more time to make sustained progress in ST Math. In shorter gameplay sessions like those found in rotation-seating, students must often quit a level and restart it later, losing all lives and progress. Children were observed to be frustrated due to losing lives or progress, and multiple teachers (H1-2, H4, M1-2, L1) were observed giving warnings several minutes before gameplay ended, specifically instructing students who made above $50 \%$ progress to continue and the rest to quit. H2, M2, and L2 appreciated that they were able to provide more help in the lab- and freeseating formats. However, two highly experienced teachers (H1, H2) believed in having a variety of formats because students learned different things from more teacher-centered and more student-centered class formats.

4.2.2 Foster Gameplay Culture. Teachers fostered a culture around ST Math, and viewed a motivating and student-centered culture as crucial to ST Math success. This is in contrast to the elaboration teacher behavior defined in Kangas et al.'s framework, where teachers frame the use of a game during each classroom session, connecting the math content to the game content. For ST Math, teachers fostered a longer-term gameplay culture, based on their own beliefs about how students learn, and how specific student groups function. Some teachers (H4, M2, L1-2) encouraged more quiet, focused, and independent work, whereas others (H1-3, M1) allowed students to talk to each other and/or move around. Some cultures were observed across classrooms. Many teachers (H1-2, M1, L1) encouraged students to share their progress on ST Math, to compete with one another, and to celebrate their achievements. To foster this culture, many teachers (H1, M1, L1) incentivized students by giving small prizes, and they perceived this technique to be effective. All teachers valued student autonomy, encouraging students to use classroom resources like manipulatives or the whiteboard and to notify teachers when they need help. H1 stated that students love ST Math due to "the environment that we have built about the importance of ST Math."

Because the county requires ST Math usage, one common factor among classrooms was a focus on making significant progress (levels completed) in ST Math. The county had previously set an expected weekly progress threshold against which teachers are evaluated based upon their students' average performance. All teachers communicated about progress with students through classroom posters and/or during gameplay. However, it was not clear if progress was a measurement that benefited students' learning, as students were observed and described to 'rush through,' resulting in low performance in the game. All teachers but L2 reported they frequently assigned objectives as homework, to give students access to gameplay at home. Teachers did so primarily to promote progress and help students catch up with the class. $\mathrm{H} 2$ mentioned an instance of healthy competition between siblings to complete ST Math at home. H2 also mentioned that by matching homework to the curriculum, parents could be involved in good conversations about math learning.

Although all teachers valued setting a classroom culture and were required to promote ST Math progress, only two highly experienced teachers $(\mathrm{H} 1, \mathrm{H} 3)$ purposely fostered a culture of playing the game with a goal of reaching "productive struggle." Productive struggle, "the kind of effortful learning that develops grit and creative problem solving," [23] is a key pedagogical goal of ST Math $[43,56]$. H1 described "never give up" as a mindset that she wanted students to have, and we observed her praising students for engaging in productive struggle. $\mathrm{H} 3$ stated that the students knew when she would not help them and instead let them solve problems by themselves. From classroom observations, it was not clear that productive struggle was sufficiently emphasized, as many students raised their hands immediately after getting wrong answers. H1, who attended MIND's PD when ST Math was first launched, wrote in her survey response that "Over the years, [the focus on productive struggle] seems to have shifted away," and expanded in her interview that many teachers "front-load the information" instead of letting students figure out how to learn from the game on their own. $\mathrm{H} 2$ commented that sometimes she "do[es] too much when we are in lab" and appreciates how the rotation-seating class format forces her to "take a step back."

4.2.3 Order the Curriculum. ST Math objectives are high level topics such as 'fractions' and 'division.' ST Math has a predefined objective sequence, but counties and schools can specify ordering based on the local math curriculum. For example, L2 stated that the school enforced some specific reorderings to ensure that difficult objectives (e.g,. fractions) were played at the same time as the concepts were taught in math class, or to ensure that they were covered before the state assessments.

Within the game, teachers have the flexibility to reorder objectives for their individual classes. We noted differences between the highly experienced and new teachers. The highly experienced teachers (H1-4) stated that they frequently reordered objectives to match what they taught in class. They believed that by practicing the same math concept twice (once in the classroom and once in ST Math), students would 
benefit from "visually understanding" and "reinforcing" what they learned in class. $\mathrm{H} 2$ stated that besides reordering "based on common sense, like what naturally builds on each other," she frequently reordered objectives to "keep it fresh" even when some students did not finish the current objective. She believed that this enabled students to "hit every strand" in ST Math without necessarily completing the curriculum, which would better prepare them for the next grade. In contrast, M1 referred to reordering as more of a school grade-level decision. M2, L1 and L2 rarely reordered with M2 stating that, "you don't want to mess it up." M1 stated that some teachers didn't know that they could reorder the objectives.

4.2.4 Connect with Classroom Content. Except for a special case of a MIND research study in H1's classroom, we did not observe teachers discussing specific game content or its purpose before or after gameplay, in contrast to the orientation and elaboration activities cited by Kangas et al. [24]. This may be because our study was conducted near the end of the year, when teachers had already finished teaching curriculum content and students were spread out in their ST Math progress. In the interviews, however, some of the more experienced teachers did reflect on using ST Math to assist their classroom teaching. Regarding introducing the game's purpose, H2 stated that during the first half of the school year, she frequently used ST Math to introduce math concepts, believing that its visual, non-verbal nature helps children understand new concepts before they must learn new vocabulary; M1 stated that she used ST Math's teacher mode to demonstrate puzzles as a whole-group activity. Regarding discussions after gameplay, $\mathrm{H} 1$ stated that she instructed the whole class or a small group on objectives where she saw "a lot of the students" struggling. H4 reflected on paying attention to students' progress in ST Math relative to their instructional progress, and took care to draw connections between the in-class math content and the topics covered in ST Math. During the discussion of game content, many teachers reflected on ST Math objectives that were difficult for many students, such as fractions (H2, H4, M2, L2) and place-value (H1, H2, H3).

4.2.5 Design Implications. Our results from interviews and classroom observations suggest that explicit supports for class formats, fostering gameplay culture, and curriculum ordering could be beneficial for curriculum-integrated games. These suggestions are in line with Dillenbourg and Jermann's theory on classroom orchestration, that one important aspect of teachers as orchestrators is planning how and when students learn within the allotted classroom formats and times [17], which as we observed, may not be under direct teacher control. Thus, teachers could benefit greatly from ST Math configurations and suggestions that support lab-, rotation-, and free-seating formats. For example, because we observed that some class formats hindered teachers' ability to pay attention to some students or to see student screens that show when students request help, we suggest ST Math provide teachers with visualization tools, such as a dashboard [16], real-time augmented reality monitoring [22], or alternative real-time communication channels such as email or text alerts when a student requests help.

Our integration results also showed that teachers were sensitive to the impact of lost progress, both due to lost lives and due to classroom time constraints. We recommend that ST Math allow students to save current progress before logging out. This could reduce teacher and student frustration when they are forced to pause due to rotations or time constraints. This is also supported by Dillenbourg and Jermann's theory that teaching time is segmented into time slices and a technology that works well must help teachers orchestrate class time [17].

Our results on fostering gameplay culture reveal that teachers were not equally prepared to support the designed intent of ST Math to promote productive struggle. However, experienced teachers in our study, and in other research [29], recognized the importance of class culture in the successful adoption of non-traditional teaching approaches. Therefore, we recommend that year-long curriculum-integrated games should explicitly build in supports that promote the target classroom culture and mindset, such as teacher guidance on setting a classroom culture, and teacher adaptable ingame awards for encouraging students to try again upon first failures. PD should also emphasize and suggest teaching practices that promote the target classroom culture and mindset. It is important to note that, in the early days of a system, the game designers and teacher professional development will naturally talk about the design rationale, but later, supports for the culture need to be built in to ensure that new community members can learn about and adopt it.

Our results suggest that only experienced teachers reorder the ST Math curriculum. Previous research shows that reordering could benefit learning, both when teachers reported doing it to tailor their classroom [11], or when it is evaluated based on student data [36]. Like ST Math, a curriculumintegrated game should be designed to have appropriate curricular sequencing, and allow teachers and districts the flexibility to adapt these sequences. On the other hand, beneficial re-orderings could be recommended within such a system. For example, Peddycord-Liu et al. found that when teachers reorder the money calculation objective before the fraction objective, students did better in fractions, which is likely due to money being a real-life example of fractions [36]. Similar systems can use such data driven insights to suggest teachers to consider reorderings when they are predicted to be beneficial. 
The lack of orientation and elaboration before and after gameplay suggests that teachers are more likely to use ST Math to conveniently assist their classroom teaching, as found in prior work [34], rather than designing teaching centered around ST Math. 'Ideal' game-based learning lessons $[18,24,57]$ that incorporate these lead-in and reflection discussions require time to plan, familiarity with the game and the curriculum, and knowing the current knowledge and skills of most students in the class. Achieving all three of these is difficult for games that are intended to be played year-long. Teachers have little time to plan or learn the game, some may be new to the curriculum and teaching, and some may not have access to appropriate PD. ST Math is also self-paced, making it difficult to gauge a whole classroom. Thus, we argue that game designers should be aware of these limitations, and design supports and PD to help teachers achieve 'ideal' integration for specific difficult concepts. Shorter, high-level introductions to specific game content within an objective, and salient mappings to the standardized curriculum content could help teachers more quickly and consistently learn about games and connect them to the classroom.

\subsection{Intervention}

Intervention is what teachers do to interact with and support students during gameplay. We identified four types of interventions from interviews and classroom observations: cognitive intervention, affective intervention, peer tutoring, and progress management. This category resembles the playing category identified by Kangas et al. [24], where teachers actively serve as tutors, facilitators, and organizers during gameplay. However, we only observed such activities with a few students at a time. We did not observe teachers pausing the game to seize 'teachable moments' for the whole class [57], probably because of the diverse activities and student progress at the time of observation. Importantly, we observed a great deal of one type of teacher activity not captured by Kangas et al.-affective intervention. During both classroom observations and interviews, teachers clearly felt that affective support was critical for grades 3-4.

Another important contribution of this work is identifying how observed intervention patterns were directly related to classroom formats. In labs, ST Math students got more help because teachers focused undivided attention on assisting students and could see on-screen indicators of student struggle. In free-seatings, ST Math's raised hand indicators frequently went unnoticed, because teachers could not easily see student screens. Teachers were also more likely to monitor and help students in large groups or near the center of the classroom. In rotation-seatings, teachers seldom intervened with the ST Math group unless those students were disruptive. Although the observers were not as familiar with the individual students' proficiency levels or personalities, we generally concluded that the students who sought help, expressed frustration verbally, or were obviously off-task, got more teacher attention than students who struggled quietly.

4.3.1 Cognitive Intervention. ST Math is specifically designed to help students learn math concepts through problem solving using visual-spatial reasoning and animated feedback. Our observations and interviews showed that teachers realized the affordances of the game and made specific efforts to help students benefit from problem solving. In noting how students could learn from ST Math, M2 stated that "it's really about if they can be observant" to ST Math's animated feedback, and teachers often reminded students to watch JiJi's animations. However, we observed that many children went off-task during the 5-10 second-long animations after each answer. H3 stated that younger children love these animations but older children just "look around." Teachers themselves stated that they needed to watch how the students had failed a couple of times before understanding how to help. Although teachers often told students that they were learning together, we did not often observe teachers explaining how they learned from watching the gameplay or animations. When we observed teachers providing help, their cognitive interventions focused on guiding questions and scaffolding by breaking problems down into smaller steps.

Teacher interviews showed that teachers recognized that learning in ST Math was more student-centered than traditional classroom teaching. Both classroom observations and interviews revealed that teachers encouraged students to find resources to support their own learning, such as consulting peers, using classroom resources such as multiplication charts, or using pencil and paper. In classrooms, we commonly observed students transferring ST Math problems into other familiar formats using classroom resources, even without teacher prompting. For example, one student was observed struggling with fractions on a numberline in ST Math; after several failures, the student decided to use paper and pencil to re-represent the fraction with a pie chart and could then progress through the level.

Classroom observations showed that children were often impatient. H2, M1, and L2 were observed telling students to "slow down and think." Students were observed ignoring animated feedback, and rushing through pre- and post-tests. Some students asked for help as soon as they got a wrong answer, with some asking teachers to "just give me the answer." Such unproductive help seeking has been observed in classrooms across contexts [3] and has also been related to the exploiting of help in intelligent tutors $[2,5]$.

4.3.2 Affective Intervention. We observed that children frequently needed help in regulating emotions during gameplay. We often observed frustration leading to off-task behaviors, 
some of which disturbed classroom activities. In teacher interviews, all teachers mentioned frustration when describing student gameplay. Many teachers (H4, M1-2, L2) stated that it was difficult for children to work out frustration on their own. Teachers (H2, H4, M1, L1) stated that children at times said "the computer is tricking me" and they needed to explain that "JiJi is a very precise penguin." Children were also afraid of JiJi dying, as observed across classrooms and heard from interviews. For example, H4 intervened with a student stating, "what happens if Jiji dies? Nothing! You get to try this again!"

From observations and interviews, it was clear that teachers believed in the importance of affective support, helping students regulate their emotions so they can benefit from the game. Teachers stated the need to distinguish between students who needed cognitive and affective support. Affective help was commonly used for students who needed "attention," a "security blanket," "a constant reminder to stay focused," or were too frustrated to proceed. Teachers also intervened by physically moving next to students without talking. We observed teachers H1, H3-4 and M1 helping frustrated students by enabling "teacher mode," on the student's computer so the student could attempt a puzzle as many times as needed without losing lives. Teachers H1H3 allowed students to replay previously-passed levels [31], stating that this was partially to alleviate student frustration with the current level. Another commonly-observed affective intervention was praising student progress. H1-2 and M1 also considered ST Math itself as an affective support to help English language learners gain confidence in math.

4.3.3 Peer Tutor. All teachers but L1 stated that they encouraged peer tutoring. Peer tutoring was observed in the classes of H1, H3, M1, and L2 when teachers sent pre-assigned "ST Math Helper" to support other children, commonly in rotation-seatings. Peer tutoring was also observed in freeseatings where children sat in groups and helped each other. Teachers reported that they recognized that: children knew more about the game; children were more likely to understand and follow peer's instructions than teacher's; teaching helped learning; and that children build confidence through tutoring peers. Teachers purposefully selected peer tutors and guided the tutoring process. ST Math Helpers were usually children who made more progress, were more proficient in math, or those who were proficient in math but needed to build their confidence. Teachers also believed that "explaining instead of telling answers" is a skill that needed to be cultivated; students lacking such ability was the main reason L1 noted that she didn't encourage peer tutoring. However, we also observed cases in which children looked at a peer's computer to solve a puzzle. In one case, the teacher caught students who switched laptops to play games on their friend's account.

4.3.4 Progress Management. Teachers acknowledged that ST Math's self-paced structure would leave students at different levels of progress. Only H3 stated a preference to keep children at similar progress levels so they could help each other. However, teachers did intervene with students at the two extremes, those far ahead and far behind. Students who were falling behind received more interventions during gameplay. Teachers (H2-3, M1) acknowledged outside factors that influenced these students, such as schedule conflicts, limited internet access at home, or being immigrants attending school for the first time. Only $\mathrm{H} 2$ stated skipping a struggling student ahead once, noting that the student needed exposure to other objectives. For students who were too far ahead, H4 and M1 moved the challenge objective (spatial puzzles without targeting specific math concepts) forward, to stop them from playing what they hadn't learned in class. Other teachers (H2, H4, M1-2, L1) assigned alternative activities, such as code.org. Two highly experienced teachers (H2-3) commented that it was important to distinguish students who were moving quickly due to their math proficiency from "rushers," who completed objectives but had lower in-game performance. H3 sent these students back to re-do rushed objectives, and $\mathrm{H} 4$ stated that she wanted to do that. One rushing behavior observed across classrooms was that some students randomly clicked instead of thoughtfully answering the objectives' pre-post multiple choice tests, a behavior that had no impact on their measured game progress.

4.3.5 Design Implications. This intervention category highlights the need to support students and teachers during gameplay. The long-term use combined with the self-paced nature of games such as ST Math causes student differences to become more salient and difficult for teachers to manage. This increases teachers' burden to provide support to individual students, and makes it difficult for individual students to get help, especially in large classes. Thus, we suggest that such games should invest in features that provide individual interventions, and support teachers in directing their attention to students who most need support during gameplay. For example, ST Math could use in-game reward mechanisms [39] as previously suggested. Our previously-suggested feature to allow students to save the current progress, may help frustrated students take a break and refocus [47].

More specific to ST Math, our results suggest new features to help students access spatial-temporal feedback at low cost. After each puzzle attempt, ST Math provides an animation that demonstrates whether the student answer solves the problem. When teachers come to help, they often need to deduce what was done by observing students fail. However, incorrect answers cause students to lose lives 
and progress. This causes some students to avoid attempting puzzles even if they could learn from the resulting animation, and some teachers to front-load information about how students should play, so they can avoid losing lives. Thus, to promote learning from failure, ST Math could allow students and teachers to replay the animations from previous attempts, and pause the animation to discuss.

Observations and interviews revealed that students often ignore the ST Math animations that show after every answer. We suggest that designers should shorten or skip animations upon correct answers, or for students who have reached a certain proficiency. This would help direct student attention to animations when they are particularly important for learning. Another related student behavior is transforming ST Math problems into other (physical) formats. This pattern is a double-edged sword: it bridges ST Math content with classroom content; however, it may not take advantage of the various game representations and scenarios designed to foster spatial-temporal reasoning [37]. It also means that game logs cannot record some learning activities, limiting the capacity of the system to learn from and adapt to student behavior. Therefore, such games could provide in-game alternative help or representations. In ST Math, explanatory animations could be improved to ensure students pay attention to them, by limiting how often they are displayed and/or requiring students to explain or interact with them [40].

Our results showed that children generally like to play together. Similar educational games should consider features such as leaderboards to foster competition while allowing children to opt-out from competition [39]. However, such features should be mindful of structuring competition that supports mastery goals [54]. Otherwise, students may rush without thinking, as we observed, or develop their own strategies to to score without necessarily learning [2, 5]. Similar ed-games should also consider features that support peer tutoring in real-time [15].

Lastly, we suggest that self-paced, large-scale games like ST Math should design specific features for students at the two progress extremes. For students far ahead of their classmates, easier access to more advanced math content was requested by teachers. For students falling behind, teachers requested a scaffolding mode or a misconception report on common wrong answers from pre-post tests. We believe it is important for long-term, curriculum-integrated games to promote equal access for all students. In the U.S., $68 \%$ of families have mobile broadband access, but only about $50 \%$ below poverty do [8]. Designers should include several alternatives, such as offline play, mobile apps, and paper worksheets.

\subsection{Data-Informed Practice}

Data-Informed Practice is what teachers do based on educational game data. This category is not explicitly identified by
Kangas et al. [24] but most relates to how teachers reflect on their practices in game-based learning. However, our results from interviews suggest teachers primarily used ST Math reports to inform teaching practices outside the game.

All teachers stated that they used ST Math reports to identify struggling students. They believed that performance in ST Math reflected students' math ability, with some exceptions due to scheduling conflicts, internet access, gaming-thesystem behavior in pre- and post-tests (H1-3), and that the language-heavy nature of non-ST Math assessments posed additional difficulties for English language learners(M1, L1). $\mathrm{M} 1, \mathrm{H} 2$, and $\mathrm{H} 4$ noted combining in-game assessments with out-of-game assessments to understand whether students were stuck on the reading comprehension elements of traditional assessments or the math concepts, and used this distinction to instruct students accordingly.

When asked how they used ST Math reports, L1 and L2 had limited understanding regarding the access and use of reports. They focused primarily on progress, with L2 requiring children to record progress on paper. Meanwhile, M1-2, and H1-4 identified both the struggling students and the content they struggled on to review and reteach in class or for specific students. $\mathrm{H} 2$ and $\mathrm{H} 4$ also cited communicating with students about what they could see in the report, to set expectations on productive learning behaviors and discourage "thinking it's just a game." H2 noted sharing good performance with parents to reward students. Such use is similar to Kangas et al.'s elaboration where teachers help students make connections between the game and learning.

4.4.1 Design Implications. Experienced teachers used ST Math reports for self-reflection, communication with students, and planning future teaching. These uses of reports support Kharrufa at al.'s findings on the kinds of information that experienced teachers would like from educational reports [29] and should be considered when designing similar reports for games. Our results also suggest that teachers, especially new teachers, need support in understanding and transforming reports into action. This suggested more accessible and integrated PD resources to support continuity in teacher's workflow [17], so teachers can see reports, read what they mean, and consider suggested actions in one place, together with clear instructions. Rather than reports geared only towards grading, curriculum-integrated games could also help teachers plan for teaching by suggesting common misconceptions or difficulties based on gameplay performance. Designers should also consider leveraging the opportunity to explore educational games' potential to assess standards-based learning from another perspective. In our study, ST Math helped teachers discern whether some students were struggling with math concepts or English reading comprehension. 


\section{WHAT DID TEACHERS SUGGEST?}

All teachers wanted a better mapping between ST Math and math standards-"if you're teaching this specific standard, these are the games you want to assign." In ST Math, objectives are mapped to standards in a specific gameplay report. However, this mapping is not visible where teachers manipulate ST Math objectives.

Teachers wanted gameplay feedback through multiple communication channels. H2 and L2 suggested post-gameplay reports via email, to check on their devices at convenient times without necessarily logging into ST Math. Teachers also expressed that current alert features were difficult to notice given limited attention and class formats. H1-2, H4, and M1-2 wanted live alerts or emails sent to their screen or phone during gameplay. For example, teachers wanted to be informed of the ST Math group's progress while instructing other groups in rotation-seating. Lastly, H2 (who displayed the report to students during gameplay) wanted the report to show positive encouraging messages, such as the increasing number of students on task.

Teachers also wanted reports to better inform their teaching. H1-3 suggested detailed answers and time taken on pre/post tests, to have a class-level summary on common misconceptions and to catch students who simply clicked through. H2-3, M1, and L2 wanted reports on estimated idle time. Teachers wanted to distinguish whether spending long amounts of time meant learning or behavior problems"either he's not getting it, or he's just not focused," and they wanted alerts for students who were significantly delayed in starting gameplay, such as doing other computer activities instead of ST Math. Moreover, when students are stuck, H1, H4, M1, and L1-2 wanted to know "what the puzzle looks like," and why they are struggling. This ties in to our suggestion that students and teachers be able to view an animation of any previously-failed puzzle attempts. M1 wanted help translating percentage progress to goals around learning of concepts, such as "I need you to master this fraction concept by the end of next week." L2 wanted the average scores of other classes, to know whether the game was difficult or she needed to teach this concept better.

Several teachers suggested making ST Math more personalized. H3 and M1 wanted access to upper-grade objectives for far-ahead students, as these students were also owed "a year of math growth" even if they started out higher performing. For far-behind students, H1 and M1 wanted to assign individualized objective orderings or homeworks without affecting the whole class. L2 suggested a "snippet" of objectives, so slower children could play a shorter version of later objectives they won't be able to get to in time before the state test. H4 and L1 suggested scaffolding some games. Other suggestions included: better PD that focuses on the propose and teaching practices of ST Math (H1, M1, L1-2) and more gamified features, such as in-game awards (M1).

Teachers' self-identified needs corresponded to our design suggestions and those from prior literature. Teachers had high expectations and hopes for the educational game- the game should make their teaching more effective, both for the whole classroom and for individual students, through conveniently-accessible information and actionable feedback from real-time alerts of students who need help, to dataderived insights for planning, to reports to inform assessment.

\section{CONCLUSION}

We have identified four categories of important teacher activities around the use of an academic year-long curriculumintegrated game in authentic classrooms: preparation, integration, intervention, and data-informed practice. We related our findings to Kangas et al.'s framework for teacher pedagogical behaviors around games in the classroom[24], identifying critical differences that arise from digital support, full academic year-long use, and curricular integration. Our results suggest considering the following features for largescale, self-paced, standards-aligned games used across the academic year:

- In-game PD, reports, and just-in-time supports for teacher workflows, to help teachers learn how and why to use the game and its data to inform teaching practices.

- Salient mappings of game content to curricula and more guidance on content reordering and manipulation.

- Support for different class formats and teacher needs for real-time feedback.

- Affective supports for individual students and designs to direct student and teacher attention to issues of particular importance.

- Supports for teachers to build and support classroom culture and social learning, such as adaptable teacher controls allowing students to collaborate, compete, and serve as peer tutors.

This research is limited by its focus on a single game, teacher selection bias, and timing of the study. While the literature supports most of our findings, future work should study whether similar issues occur for other academic yearlong, curriculum aligned games, for non-volunteer teachers, and at other times of the school year.

\section{ACKNOWLEDGEMENT}

This material is based upon work supported by the National Science Foundation under Grant No. 1544273. We would like to thank Andrea Kunze, Jennifer Tsan, Raven Robinson, Sarah Karamarkovich, and Christa Cody and the participating schools who enabled this study. 


\section{REFERENCES}

[1] Adkins, S. (2017). The 2017-2022 Global Game-based Learning Market. In Serious Play Conference.

[2] Aleven, V., McLaren, B., Roll, I., \& Koedinger, K. (2004). Toward tutoring help seeking: Applying cognitive modeling to meta-cognitive skills. In J. C. Lester, R. M. Vicari, \& F. Paraguacu, Proceedings of Seventh International Conference on Intelligent Tutoring Systems, ITS 2004 (pp. 227-239). Berlin: Springer Verlag.

[3] Arbreton, A. Student Goal Orientation and HelpSeeking Strategy Use. In S.A. Karabenick (Ed.), Strategic Help Seeking: Implications For Learning And Teaching, Lawrence Erlbaum Associates, Mahwah, NJ (1998), 95-116.

[4] Backlund, P., \& Hendrix, M. (2013, September). Educational gamesare they worth the effort? A literature survey of the effectiveness of serious games. In Games and virtual worlds for serious applications (VS-GAMES), 2013 5th international conference on (pp. 1-8). IEEE.

[5] Baker, R., Walonoski, J., Heffernan, N., Roll, I., Corbett, A., \& Koedinger, K. (2008). Why students engage in "gaming the system" behavior in interactive learning environments. Journal of Interactive Learning Research, 19(2), 185-224.

[6] Bakker, M., van den Heuvel-Panhuizen, M., \& Robitzsch, A. (2015). Effects of playing mathematics computer games on primary school students' multiplicative reasoning ability. Contemporary Educational Psychology, 40, 55-71.

[7] Barendregt, W., \& Bekker, T. M. (2011). The influence of the level of free-choice learning activities on the use of an educational computer game. Computers \& Education, 56(1), 80-90.

[8] Bauman, K. (2018). More than Two-Thirds Access Internet on Mobile Devices. In America Counts: Stories: Population. U.S. Census Bureau. August 8, 2018. Online Jan. 4, 2019: https://www.census.gov/library/stories/2018/08/internet-access.html

[9] Boyle, E. A., Hainey, T., Connolly, T. M., Gray, G., Earp, J., Ott, M., ... \& Pereira, J. (2016). An update to the systematic literature review of empirical evidence of the impacts and outcomes of computer games and serious games. Computers \& Education, 94, 178-192.

[10] Buabeng-Andoh, C. (2012). Factors Influencing Teachers' Adoption and Integration of Information and Communication Technology into Teaching: A Review of the Literature. International Journal of Education and Development using Information and Communication Technology, 8(1), 136-155.

[11] Callaghan, M. N., Long, J. J., van Es, E. A., Reich, S. M., \& Rutherford, T. (2018). How teachers integrate a math computer game: Professional development use, teaching practices, and student achievement. Journal of Computer Assisted Learning, 34(1), 10-19.

[12] Chee, Y. S., Mehrotra, S., \& Ong, J. C. (2014). Facilitating dialog in the game-based learning classroom: Teacher challenges reconstructing professional identity.

[13] Clark, D. B., Tanner-Smith, E. E., \& Killingsworth, S. S. (2016). Digital games, design, and learning: A systematic review and meta-analysis. Review of educational research, 86(1), 79-122.

[14] Demirbilek, M., \& Tamer, S. L. (2010). Math teachers' perspectives on using educational computer games in math education. Procedia-Social and Behavioral Sciences, 9, 709-716.

[15] Diana, N., Eagle, M., Stamper, J., Grover, S., Bienkowski, M., \& Basu, S. (2017, March). An instructor dashboard for real-time analytics in interactive programming assignments. In Proceedings of the Seventh International Learning Analytics \& Knowledge Conference (pp. 272279). ACM

[16] Diana, N., Eagle, M., Stamper, J., Grover, S., Bienkowski, M., \& Basu, S. (2017, March). An instructor dashboard for real-time analytics in interactive programming assignments. In Proceedings of the Seventh
International Learning Analytics \& Knowledge Conference (pp. 272279). ACM.

[17] Dillenbourg, P., \& Jermann, P. (2010). Technology for classroom orchestration. In New science of learning (pp. 525-552). Springer, New York, NY.

[18] Eastwood, J. L., \& Sadler, T. D. (2013). Teachers' implementation of a game-based biotechnology curriculum. Comp. \& Ed., 66, 11-24.

[19] Girotto, V., Lozano, C., Muldner, K., Burleson, W., \& Walker, E. (2016, May). Lessons learned from in-school use of rtag: A robo-tangible learning environment. In Proceedings of the 2016 CHI Conference on Human Factors in Computing Systems (pp. 919-930). ACM.

[20] Hebert, C., \& Jenson, J. (2017). Digital Game-Based Pedagogy: Exploring Teaching Strategies for Classroom Teachers in the use of Video Games in K-12 Classrooms. In ECGBL 2017 11th European Conference on Game-Based Learning (p. 227).

[21] Hieftje, K., Pendergrass, T., Kyriakides, T. C., Gilliam, W., \& Fiellin, L. (2017). An Evaluation of an Educational Video Game on Mathematics Achievement in First Grade Students. Technologies, 5(2), 30.

[22] Holstein, K., Hong, G., Tegene, M., McLaren, B. M., \& Aleven, V. (2018, March). The classroom as a dashboard: co-designing wearable cognitive augmentation for K-12 teachers. In Proceedings of the 8th International Conference on Learning Analytics and Knowledge (pp. 79-88). ACM.

[23] Kang, H. 8 Teaching Habits that Block Productive Struggle in Math Students. Retrieved September 10, 2018, from https://blog.mindresearch.org/blog/productive-struggle-in-math

[24] Kangas, M., Koskinen, A., \& Krokfors, L. (2017). A qualitative literature review of educational games in the classroom: the teacher's pedagogical activities. Teachers and Teaching, 23(4), 451-470.

[25] Ke, F. (2006). Classroom goal structures for educational math game application. In Proceedings of the 7 th international conference on Learning sciences (pp. 314-320). International Society of the Learning Sciences

[26] Ke, F. (2011). A qualitative meta-analysis of computer games as learning tools. In Gaming and Simulations: Concepts, Methodologies, Tools and Applications (pp. 1619-1665). IGI Global.

[27] Kiili, K., \& Ketamo, H. (2018). Evaluating cognitive and affective outcomes of a digital game-based math test. IEEE Transactions on Learning Technologies, 11(2), 255-263.

[28] King, A. (2011). Using interactive games to improve math achievement among middle school students in need of remediation (Doctoral dissertation, The George Washington University).

[29] Kharrufa, A., Rix, S., Osadchiy, T., Preston, A., \& Olivier, P. (2017, May). Group Spinner: recognizing and visualizing learning in the classroom for reflection, communication, and planning. In Proceedings of the 2017 CHI Conference on Human Factors in Computing Systems (pp. 5556-5567). ACM Computing, 16, 1-14.

[30] Lim, C. P., Tay, L. Y., \& Hedberg, J. (2011). Employing an activitytheoretical perspective to localize an educational innovation in an elementary school. Journal of Educational Computing Research, 44(3), 319-344.

[31] Liu, Z., Cody, C., Barnes, T., Lynch, C., and Rutherford, T. (2017). The Antecedents of and Associations with Elective Replay in An Educational Game: Is Replay Worth It. In proceedings of the 10th International Conference on Educational Data Mining (EDM).

[32] Mifsud, C. L., Vella, R., \& Camilleri, L. (2013). Attitudes towards and effects of the use of video games in classroom learning with specific reference to literacy attainment. Research in Education, 90(1), 32-52.

[33] Molin, G. (2017). The role of the teacher in game-based learning: A review and outlook. In Serious Games and Edutainment Applications (pp. 649-674). Springer, Cham.

[34] Nanavati, A., Dias, M. B., \& Steinfeld, A. (2018, April). Speak Up: A Multi-Year Deployment of Games to Motivate Speech Therapy in India. 
In Proceedings of the 2018 CHI Conference on Human Factors in Computing Systems (p. 318). ACM.

[35] Niederhauser, D. S., \& Stoddart, T. (2001). Teachers' instructional perspectives and use of educational software. Teaching and teacher education, 17(1), 15-31.

[36] Peddycord-Liu, Z., Cody, C., Kessler, S., Barnes, T., Lynch, C. F., and Rutherford, T. (2017, October). Using Serious Game Analytics to Inform Digital Curricular Sequencing: What Math Objective Should Students Play Next?. In proceedings of the Annual Symposium on ComputerHuman Interaction in Play (pp. 195-204). ACM.

[37] Peddycord-Liu, Z., Harred, R., Karamarkovich, S., Barnes, T., Lynch, C., \& Rutherford, T. (2018, June). Learning Curve Analysis in a Large-Scale, Drill-and-Practice Serious Math Game: Where Is Learning Support Needed? In International Conference on Artificial Intelligence in Education (pp. 436-449). Springer, Cham.

[38] Peddycord-Liu, Z. A. (2018). Game Learning Analytics and Qualitative Methods for Actionable Change in a Curriculum-Integrated Educational Math Game. (Doctoral dissertation, North Carolina State University).

[39] Ravyse, W. S., Blignaut, A. S., Leendertz, V., \& Woolner, A. (2017). Success factors for serious games to enhance learning: a systematic review. Virtual Reality, 21(1), 31-58.

[40] Rau, M. A., Aleven, V., \& Rummel, N. (2009, July). Intelligent Tutoring Systems with Multiple Representations and Self-Explanation Prompts Support Learning of Fractions. In AIED (pp. 441-448).

[41] Richards, J., Stebbins, L., \& Moellering, K. (2013). Games for a digital age: K-12 market map and investment analysis. In Joan Ganz Cooney Center at Sesame Workshop, New York.

[42] Riconscente, M. M. (2013). Results from a controlled study of the iPad fractions game Motion Math. Games and Culture, 8(4), 186-214.

[43] Roll, I., Baker, R. S. D., Aleven, V., \& Koedinger, K. R. (2014). On the benefits of seeking (and avoiding) help in online problem-solving environments. Journal of the Learning Sciences, 23(4), 537-560.

[44] Rowe, E., Asbell-Clarke, J., Baker, R. S., Eagle, M., Hicks, A. G., Barnes, T. M., ... \& Edwards, T. (2017). Assessing implicit science learning in digital games. Computers in Human Behavior, 76, 617-630.

[45] Rutherford, T., Kibrick, M., Burchinal, M., Richland, L., Conley, A., Osborne, K., ... \& Daniels, A. (2010). Spatial Temporal Mathematics at Scale: An Innovative and Fully Developed Paradigm to Boost Math Achievement among All Learners. Available from ERIC database (ED510612).
[46] Rutherford, T., Farkas, G., Duncan, G., Burchinal, M., Kibrick, M., Graham, J., ... \& Martinez, M. E. (2014). A randomized trial of an elementary school mathematics software intervention: Spatial-temporal math. Journal of Research on Educational Effectiveness, 7(4), 358-383.

[47] Sabourin, J., Rowe, J. P., Mott, B. W., \& Lester, J. C. (2011, June). When off-task is on-task: The affective role of off-task behavior in narrativecentered learning environments. In International Conference on Artificial Intelligence in Education (pp. 534-536). Springer, Berlin, Heidelberg.

[48] Sadler, T. D., Romine, W. L., Stuart, P. E., \& MerleâĂǨJohnson, D. (2013). GameâĂŘbased curricula in biology classes: Differential effects among varying academic levels. Journal of Research in Science Teaching, 50(4), 479-499.

[49] Saldana, J. (2015). The coding manual for qualitative researchers. Sage.

[50] Sandford, R., Ulicsak, M., Facer, K., \& Rudd, T. (2006). Teaching with games: Using commerical off-the-shelf computer games in formal education. Nesta Future Lab. Last retrieved online at:https://www.nfer.ac.uk/publications/FUTL49/FUTL49.pdf

[51] Schenke, K., Rutherford, T., \& Farkas, G. (2014). Alignment of game design features and state mathematics standards: Do results reflect intentions?. Computers \& Education, 76, 215-224.

[52] Speak, U. P. From Print to Pixel: The role of videos, games, animations and simula-tions within K-12 education.http://www.tomorrow.org/speakup/SU15AnnualReport.html. 2015.

[53] Takeuchi, L. M., \& Vaala, S. (2014). Level up Learning: A National Survey on Teaching with Digital Games. Joan Ganz Cooney Center.

[54] Urdan, T., \& Schoenfelder, E. (2006). Classroom effects on student motivation: Goal structures, social relationships, and competence beliefs. Journal of school psychology, 44(5), 331-349.

[55] van der Maas, H. L., \& Nyamsuren, E. (2017). Cognitive analysis of educational games: The number game. Topics in cognitive science, 9(2), 395-412.

[56] Warshauer, H. K. (2015). Productive struggle in middle school mathematics classrooms. Journal of Mathematics Teacher Education, 18(4), 375-400.

[57] Watson, W. R., Mong, C. J., \& Harris, C. A. (2011). A case study of the inclass use of a video game for teaching high school history. Computers \& Education, 56(2), 466-474.

[58] Watson, W., \& Yang, S. (2016). Games in schools: Teachers' perceptions of barriers to game-based learning. Journal of Interactive Learning Research, 27(2), 153-170. 